\title{
(2) OPEN ACCESS \\ Characteristics of patients who had a stroke not initially identified during emergency prehospital assessment: a systematic review
}

\author{
Stephanie P Jones (10 , 'Janet E Bray, ${ }^{2} J o s e p h i n e ~ M E ~ G i b s o n,{ }^{1}$ \\ Graham McClelland 자, ${ }^{3,4}$ Colette Miller, ${ }^{1}$ Chris I Price (1), ${ }^{4}$ Caroline L Watkins ${ }^{1}$
}

Handling editor Loren De Freitas

Additional material is published online only. To view, please visit the journal online (http://dx.doi.org/10.1136/ emermed-2020-209607).

${ }^{1}$ Faculty of Health and Care, University of Central Lancashire, Preston, UK

${ }^{2}$ Department of Epidemiology and Preventive Medicine,

Monash University, Melbourne, Victoria, Australia

${ }^{3}$ Research and Development North East Ambulance Service NHS Foundation Trust, Newcastle Upon Tyne, UK ${ }^{4}$ Institute of Neuroscience (Stroke Research Group), Newcastle University, Newcastle upon Tyne, UK

\section{Correspondence to}

Stephanie P Jones, Faculty of Health and Care, University of Central Lancashire, Preston PR1 2HE, UK; sjones10@uclan.ac.uk

Received 18 April 2020 Revised 30 October 2020 Accepted 2 December 2020 Published Online First 19 February 2021

\section{ABSTRACT \\ Background Around $25 \%$ of patients who had a} stroke do not present with typical 'face, arm, speech' symptoms at onset, and are challenging for emergency medical services (EMS) to identify. The aim of this systematic review was to identify the characteristics of acute stroke presentations associated with inaccurate EMS identification (false negatives).

Method We performed a systematic search of MEDLINE, EMBASE, CINAHL and PubMed from 1995 to August 2020 using key terms: stroke, EMS, paramedics, identification and assessment. Studies included: patients who had a stroke or patient records; $\geq 18$ years; any stroke type; prehospital assessment undertaken by health professionals including paramedics or technicians; data reported on prehospital diagnostic accuracy and/ or presenting symptoms. Data were extracted and study quality assessed by two researchers using the Quality Assessment of Diagnostic Accuracy Studies V.2 tool. Results Of 845 studies initially identified, 21 observational studies met the inclusion criteria. Of the 6934 stroke and Transient Ischaemic Attack patients included, there were 1774 (26\%) false negative patients (range from $4(2 \%)$ to $247(52 \%))$. Commonly documented symptoms in false negative cases were speech problems ( $n=107 ; 13 \%-28 \%)$, nausea/vomiting $(n=94 ; 8 \%-38 \%)$, dizziness $(n=86 ; 23 \%-27 \%)$, changes in mental status $(n=51 ; 8 \%-25 \%)$ and visual disturbance/impairment ( $n=43 ; 13 \%-28 \%$ ).

Conclusion Speech problems and posterior circulation symptoms were the most commonly documented symptoms among stroke presentations that were not correctly identified by EMS (false negatives). However the addition of further symptoms to stroke screening tools requires valuation of subsequent sensitivity and specificity, training needs and possible overuse of high priority resources.

\section{BACKGROUND}

Worldwide, each year approximately 20 million people experience a stroke, of whom 5 million will die and 5 million will be disabled by their stroke. ${ }^{1}$ Accurate, early recognition is necessary to maximise benefits of hyperacute treatment with intravenous thrombolysis and/or mechanical thrombectomy, where indicated and early specialist multidisciplinary care. ${ }^{2}$ With up to $70 \%$ of patients who had a stroke accessing the emergency medical services (EMS), ${ }^{4}$ the efficiency of the 'stroke chain of survival' relies heavily on the accuracy and

\section{Key messages}

What is already known on this subject?

- The sensitivity of prehospital screening tools for strokes ranges from $44-97 \%$, partly due to the fact that about $25 \%$ of patients present with less common stroke symptoms.

- Prehospital recognition of stroke can be challenging. There is currently no consensus about whether to screen for additional symptoms, and which symptoms should be included in this expanded screening.

What this study adds?

- In this systematic review of studies on prehospital stroke recognition, between $2 \%$ and $52 \%$ of all stroke presentations transported by emergency medical services are not identified in the prehospital setting.

- Amongst stroke presentations that are not correctly identified by the EMS (false negatives) speech problems and posterior circulation symptoms including: nausea/vomiting, dizziness and visual disturbance/impairment were the most commonly documented characteristics.

- As some of these symptoms are very nonspecific, adding further symptoms to stroke screening tools requires further evaluation of sensitivity and specificity, training needs, and possible overuse of high priority resources

timeliness of EMS identification of stroke symptoms and the ability to distinguish between stroke and non-stroke cases.

The use of screening tools to identify stroke by the EMS is recommended internationally including in guidelines for Australia, New Zealand, Europe and the USA. The majority of prehospital screening tools feature assessments for the most common stroke symptoms, as first reported in the Cincinnati Prehospital Stroke Scale (CPSS), also known as the Face Arm Speech Test (FAST). ${ }^{5}$ However, the accuracy of prehospital screening tools varies: sensitivity is reported ranging from $44 \%$ to $97 \%$ and specificity from $13 \%$ to $92 \% .^{6}$ The diverse nature of less common stroke symptoms such as visual disturbance, confusion and loss of balance can make correct identification challenging, particularly as up to $25 \%$ of patients who had a stroke 
do not present with symptoms commonly featured in screening tools. $^{7}$

To date, there has not been an overview describing which symptoms are most common among patients who are not identified by the EMS, and there is currently no consensus about whether to assess symptoms with reduced specificity for stroke. Without screening tools and training to improve the identification of patients with less common stroke symptoms, inequity of available stroke care for patients will remain, particularly for patients with posterior stroke. ${ }^{8}$ The aim of this systematic review was to identify the characteristics of acute stroke presentations associated with inaccurate EMS identification (false negatives). Research objectives were to identify what proportion of patients who had a stroke are not identified by EMS/prehospital tools, to examine any differences in outcomes between false negative cases and those which are correctly identified, and to explore which symptoms are most commonly present in false negative cases.

\section{METHODS}

\section{Search strategy and study selection}

A search strategy was developed (online supplemental file 1), including the Medical Subject Heading terms stroke, EMS, paramedics, recognition and screening. The search strategy was adapted to search MEDLINE, EMBASE, CINAHL and PubMed from 1995 to August 2020. Studies were included from any country if published in English, with no restrictions on study design or quality.

Inclusion criteria: studies including patients who had a stroke (either actual patients $\geq 18$ years or their records, any stroke type); studies including patients screened by health professionals including paramedics or technicians within the prehospital setting; data reported on prehospital diagnostic accuracy and/or symptoms present.

Exclusion criteria: non-stroke populations, studies including only stroke mimics, studies utilising prehospital screening tools to identify large vessel occlusion.

\section{Review methods}

Citations were screened independently by two researchers on title and then abstract. Any articles that met the inclusion criteria were read in full. Disagreements over the inclusion of any articles were discussed by members of the project steering group (SPJ, JMEG and CM). Backward and forward citation searches were performed to identify further studies and to test the quality of the search strategy.

\section{Assessment of risk of bias in included studies}

Study quality was assessed using the Quality Assessment of Diagnostic Accuracy Studies V.2 (QUADAS-2) tool, ${ }^{9}$ comprising four domains: patient selection, index test, reference standard and flow and timing. We added the signalling question 'is data collected prospectively or retrospectively' within the patient selection domain. Any retrospective studies were categorised as high risk for the patient selection domain.

\section{Data extraction and management}

We designed a data extraction form that summarised the following characteristics: (1) Study detail (author, year of publication, study type, screening completed by, timing of data collection, screening tool used); (2) Patient characteristics (population, sample size, age, sex, stroke type, signs and symptoms of patients missed by the EMS recorded in prehospital and/or hospital

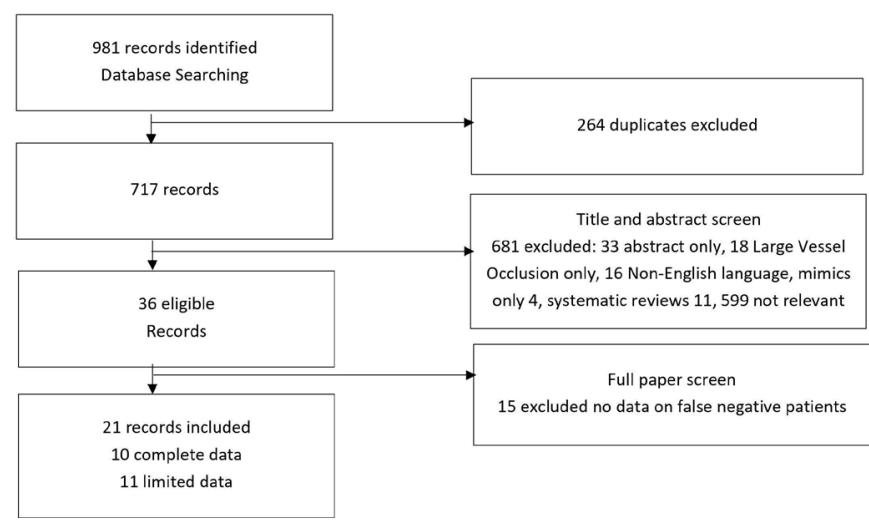

Figure 1 Flow diagram.

records, hospital diagnosis of stroke); and (3) Study quality (patient selection, risk of bias and applicability).

The accuracy of data extraction was checked by a second independent extractor for all included studies. We contacted study authors for missing data but at the time of writing had not received any responses. The protocol for the review was registered on PROSPERO. ${ }^{10}$ The reporting of this review is in accordance with the Preferred Reporting Items for Systematic Reviews and Meta-Analyses. ${ }^{11}$

\section{Analysis}

A priori it had been intended to perform a meta-analysis but due to heterogeneity between study settings, designs and screening tools used, the included studies have been described narratively. Results are reported as presented in the original studies, and no additional secondary analyses have been undertaken.

\section{Results}

The search strategy initially identified 845 articles. Following screening of the title, abstract or complete article, 21 studies met the inclusion criteria (see figure 1). Across all 21 studies, the number of included stroke patients totalled 6934, ranging from 35 to 997 . Studies took place in the following countries: 10 in the $\mathrm{USA}^{512-20} ; 3$ in the $\mathrm{UK}^{21-23} ; 3$ in Australia ${ }^{24-26} ; 2$ in China, ${ }^{27} 28$ 2 in Sweden ${ }^{29} 30$ and 1 in Canada. ${ }^{31}$ Of the 21 included studies, 11 reported limited data and included no information on age, sex or symptoms.

\section{Study quality}

The studies' overall quality can be seen in online supplemental file 2. Six studies were identified as having a low risk of bias across 4 domains of the QUADAS-2,12 $2426-2831$ although only four reported symptom data. ${ }^{1242627}$ The majority of studies had a low risk of bias in terms of the screening tool used, confirmed diagnosis of stroke or non-stroke, flow and timing of emergency screening and final diagnosis. Fourteen studies had a high risk of selection bias, 12 due to retrospective designs ${ }^{13-18} 222326$ 29-31; others due to select patient groups including: only patients who were transported to a specialist centre, ${ }^{21}$ participants defined by paramedic impression only ${ }^{19}$ and a convenience sample of patients presenting to the ED or inpatient neurology services. ${ }^{5}$

\section{False negative cases}

In all 21 studies, the number of false negative patients totalled $1774(26 \%)$, ranging from $4(2 \%)$ to $247(52 \%)$. Only 10 of the 21 studies reported any further patient information. Details of the 10 studies with presentation of data describing a complete 


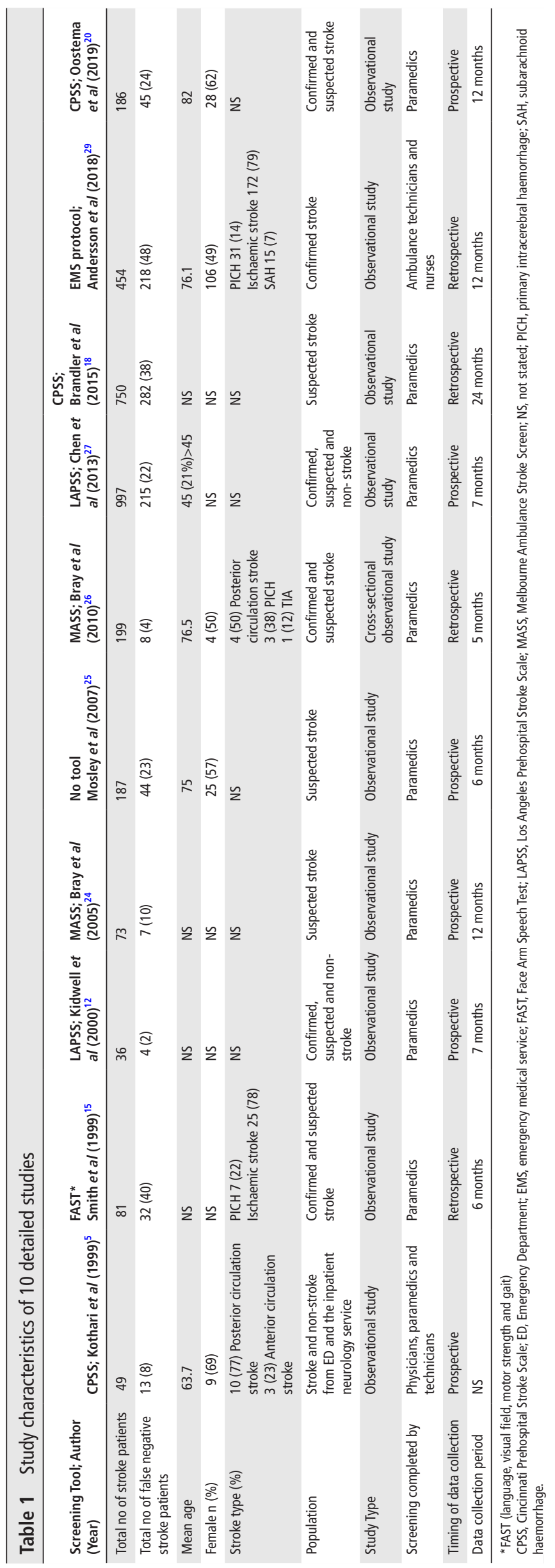

suspected stroke cohort are summarised in table 1 . Symptom data are presented in table 2 and details of the remaining 11 studies in table 3. Of these 10 studies, the number of included stroke patients totalled 3012 of whom 868 (29\%) were false negative, ranging from $4(2 \%)$ to $282(38 \%)$. Only five studies reported mean age or sex: in these studies, the mean age was 74.7 years and $57 \%$ of participants were female. Four studies reported specific stroke types, with the majority of false negative patients having ischaemic strokes, followed by primary intracerebral haemorrhage $(41,15 \%)$ and subarachnoid haemorrhage $(15,6 \%)$.

\section{Use of prehospital screening tools}

A range of stroke screening tools were used: the CPSS (five studies) ${ }^{5} 131418{ }^{20}$; the FAST (three studies) ${ }^{22}{ }^{23}{ }^{30}$; the Los Angeles Prehospital Stroke Scale (LAPSS) (two studies) ${ }^{12} 27$; the Melbourne Ambulance Stroke Screen (two studies). ${ }^{24}{ }^{26}$ One study used each of the following: Cincinnati Stroke Triage Assessment $\mathrm{Too}^{19}$; the Ontario Prehospital Stroke Screening $\mathrm{Too}^{31}$; LAPSS and CPSS ${ }^{17}$; Medic Prehospital Assessment for Code Stroke and CPSS ${ }^{16}$; Recognition of Stroke in the Emergency Room (ROSIER) and FAST ${ }^{21}$; ROSIER and CPSS. ${ }^{28}$

\section{Symptoms experienced by the false negative stroke patients}

From the data available, it was not possible to determine whether symptoms were recorded by the EMS or identified later in hospital. In 10 studies reporting symptom data for false negative patients, the most commonly recorded were: speech problems $(\mathrm{n}=107 ; 13 \%-28 \%)^{20252629}$; nausea/vomiting $(\mathrm{n}=94 ; 8 \%-38 \%)^{18202629}$; dizziness $(\mathrm{n}=86 ; 23 \%-27 \%)^{5182026}$; visual disturbance/impairment (visual loss, diplopia or blurring) $(\mathrm{n}=43 ; 13 \%-29 \%)^{5202429}$ and changes in mental status $(\mathrm{n}=51$; 8\%-25\%). 515182026

\section{Acute clinical outcomes in false negative cases}

Of the 21 studies in total, only 8 (38\%) reported any information in relation to management and treatment pathways. Five of these were undertaken between 1997 and 2009,5122631 all of which stated that false negative patients had minimal or atypical symptoms and would not have been candidates for thrombolysis based on protocols at the time. Three further studies took place between 2010 and 2019..20 2329 In these studies, EMS-recognised strokes had significantly faster door-to-CT times $(34.6$ vs $84.7 \mathrm{~min} ; \mathrm{p}<0.001)$, but this did not translate into significantly higher rates of thrombolysis delivery $(14.9 \%$ vs $4.4 \% ; \mathrm{p}=0.074)$. When patients were FAST-positive or a prealert was made, the median time from hospital arrival to CT request and scan was 39 and $57 \mathrm{~min}$ and 26 and $39 \mathrm{~min}$, respectively, compared with medians of 120 and $155 \mathrm{~min}$ for FAST negative patients and 125 and $185 \mathrm{~min}$ for patients arriving at hospital without a prealert. ${ }^{23}$ One study reported that a pre-alert was made for only $11 \%$ of patients who had a stroke who were not identified by the EMS compared with $70 \%$ of stroke patients who were identified $(\mathrm{p}<0.001) .{ }^{29}$ Patients for whom the EMS did not identify stroke nor pre-alert the receiving centre had the longest time from ambulance call to first medical assessment in the Emergency Department (ED) at $87 \mathrm{~min}(68-147)$ and $52 \mathrm{~min}$ $(45-73)$, respectively. ${ }^{25}$

\section{DISCUSSION}

This is the first review that has systematically synthesised the research evidence identifying the signs and symptoms of patients who had a stroke who were not initially identified by the EMS. 
Table 2 Frequency (\%) of all symptoms reported for false negative stroke patients

\begin{tabular}{|c|c|c|c|c|c|c|c|c|c|c|}
\hline & $\begin{array}{l}\text { Kothari et al } \\
(1999)^{5}\end{array}$ & $\begin{array}{l}\text { Smith et al } \\
(1999)^{15}\end{array}$ & $\begin{array}{l}\text { Kidwell et al } \\
(2000)^{12}\end{array}$ & $\begin{array}{l}\text { Bray et al } \\
(2005)^{24}\end{array}$ & $\begin{array}{l}\text { Mosley et al } \\
(2007)^{25}\end{array}$ & $\begin{array}{l}\text { Bray et al } \\
(2010)^{26}\end{array}$ & $\begin{array}{l}\text { Chen et al } \\
(2013)^{27}\end{array}$ & $\begin{array}{l}\text { Brandler et } \\
\text { al }(2015)^{18}\end{array}$ & $\begin{array}{l}\text { Andersson et } \\
\text { al }(2018)^{29}\end{array}$ & $\begin{array}{l}\text { Oostema et } \\
\text { al }(2019)^{20}\end{array}$ \\
\hline \multicolumn{11}{|l|}{ Symptoms n (\%) } \\
\hline Facial droop & - & - & - & - & $4(9)$ & - & - & - & $27(12)$ & - \\
\hline Arm weakness/drift & - & - & - & - & $14(32)$ & - & - & - & - & - \\
\hline Leg weakness/drift & - & - & $1(25)$ & - & - & - & - & - & - & - \\
\hline Arm/leg weakness/drift & - & - & - & - & - & - & - & - & $69(32)$ & - \\
\hline Facial droop or arm weakness & - & - & - & - & - & - & $81(38)$ & - & - & - \\
\hline Speech problems & - & - & - & - & $10(23)$ & $1(13)$ & - & - & $61(28)$ & $35(78)$ \\
\hline Visual disturbance/impairment & $2(15)$ & - & - & $2(29)$ & - & - & - & & $28(13)$ & $11(6)$ \\
\hline Ataxia & $3(23)$ & - & - & - & - & $2(25)$ & - & & - & $13(30)$ \\
\hline Dizziness & $3(23)$ & - & - & - & - & $2(25)$ & - & $77(27)$ & - & $4(9)$ \\
\hline Vertigo & $3(23)$ & - & - & - & -- & - & - & & $96(44)$ & $7(16)$ \\
\hline Nausea or vomiting & - & - & - & - & - & $3(38)$ & - & $22(8)$ & $64(29)$ & $5(12)$ \\
\hline Sensory deficit & - & - & - & - & - & - & - & & - & - \\
\hline Headache & - & - & - & - & - & $1(13)$ & - & $40(14)$ & $40(18)$ & $9(21)$ \\
\hline Unilateral weakness & $4(31)$ & - & - & - & - & - & - & & - & $22(48.9)$ \\
\hline Mental status changes & $1(8)$ & $6(19)$ & - & - & - & $2(25)$ & - & $34(12)$ & - & $8(19)$ \\
\hline Change in conscious level & - & $2(6)$ & - & - & - & $1(13)$ & - & - & - & - \\
\hline Hypoglycaemia & - & - & - & $1(14)$ & - & - & - & - & - & - \\
\hline Seizure & - & $2(6)$ & - & - & - & - & - & $12(4)$ & - & - \\
\hline Quadriparesis & - & - & - & - & - & - & - & $6(2)$ & - & - \\
\hline Bilateral weakness & - & - & $2(50)$ & - & - & - & - & - & - & - \\
\hline Weakness other & - & - & - & - & - & - & -- & $91(32)$ & - & - \\
\hline Ophthalmoplegia & - & - & $1(25)$ & - & - & - & - & - & - & - \\
\hline Fever & - & - & - & - & - & $1(13)$ & - & - & - & - \\
\hline Incontinence & - & - & - & - & - & $2(25)$ & - & - & - & - \\
\hline Fall & - & - & - & - & - & $2(25)$ & - & - & - & - \\
\hline
\end{tabular}

Across 21 studies, 26\% of patients who had a stroke were not recognised by the EMS, ranging from between $2 \%$ and $52 \%$ of stroke presentations not identified in the prehospital setting. It should be noted that study quality and size varied considerably, with even studies using the same screening tools reporting substantial differences in the proportion of false negative patients. $^{1314}$

EMS identification of patients who had a stroke enables patients to access the stroke pathway at the earliest opportunity, which expedites, where indicated, a prealert to the receiving hospital and subsequent transfer to a specialist centre. Research suggests that patients who had a stroke who are prealerted to the receiving hospital have significantly reduced times from onset to hospital arrival and specialist assessment, leading to higher thrombolysis rates and better outcomes. ${ }^{23}$

Although a total of 30 different stroke symptoms were reported across 10 studies, the most common symptoms among false negative patients were speech problems, nausea/vomiting, dizziness, changes in mental status and visual disturbance/impairment. While in some cases, individual patient presentations can hinder assessment, it is surprising that patients who had a stroke with speech problems are so often misidentified by the EMS, especially given that speech problems are the most commonly reported stroke symptom of patients and callers to the EMS for suspected stroke. ${ }^{2632} 33$ In studies using prehospital screening tools, the majority of tools, including the widely used FAST test, include assessment of speech (excluding LAPSS and C-STAT). It may be challenging to identify milder speech problems, especially in patients presenting with confusion or where the history is not clear, in the prehospital setting. It is also possible that for some patients in the included studies, their speech problems were not present on initial assessment and evolved only after hospital admission.

Nausea/vomiting occurs in around $20 \%$ of acute stroke patients, most frequently in those with vertebrobasilar stroke. One ambulance service in the UK has recently added nausea/ vomiting to their prehospital screening tool for stroke, which also includes vertigo, visual problems and ataxia. ${ }^{34}$ The impact of this on the specificity of EMS stroke identification is unknown but may be considerable given that nausea/vomiting is a common symptom across a range of acute illnesses.

Dizziness is one of the most commonly reported symptoms in cerebellar stroke, occurring in up to three-quarters of patients. The term dizziness is non-specific but may be used to describe vertigo and presyncope. Although other focal neurological symptoms may accompany dizziness, dizziness alone presents in fewer than $1 \%$ of all patients who had a stroke. ${ }^{35}$ In a recent retrospective analysis of National Institutes of Health Stroke Scale data, the addition of balance (defined as gait imbalance or leg weakness) and visual symptoms (visual loss and diplopia) to FAST symptoms would have improved recognition of stroke from $86 \%$ to $96 \%(\mathrm{p}<0.0001){ }^{36}$ Similarly, in another study, the addition of ataxia or visual symptoms to the FAST would have increased sensitivity from $61 \%$ to $80 \%(\mathrm{p}<0.001)$ and $82 \%$ $(\mathrm{p}<0.001)$, respectively ${ }^{37}$; and in a further study of patients with posterior circulation stroke, FAST combined with ataxia and visual disturbance or blindness would have improved sensitivity from $70 \%$ to $84 \% .^{22}$ However, these studies preclude any estimate of specificity because they were limited to patients with confirmed stroke 223637 ; further, sample sizes were small ${ }^{23}$ and retrospective designs were used. ${ }^{22}{ }^{37}$ In a further study aiming to increasing sensitivity to posterior circulation stroke, balance and 
eyes were added to the FAST (BEFAST). The Balance component of the BEFAST scale was scored by finger-to-nose testing and the Eyes component by assessing for diplopia using finger tracking. However, the addition of these additional symptoms did not improve stroke recognition. ${ }^{38}$ Stroke-related visual problems also occur commonly during posterior circulation stroke but are challenging to recognise for both health professionals and patients and only around $20 \%$ of patients who had a stroke presenting predominantly with visual symptoms contact the EMS. While EMS identification of visual disturbance/impairment may be feasible, agreement would be needed around which visual problems should be assessed, how and by whom.

Five studies reported changes in mental status, ranging from $8 \%$ to $25 \% .^{5} 15182026$ Mental status was largely undefined in these studies but may include a range of symptoms: confusion, delirium, altered orientation and memory are more common in older patients, those with pre-existing cognitive impairments and underlying infections. While changes in mental status occur in up to one-third of patients who had a stroke, stroke is a rare cause $(<3 \%)$ of isolated changes in mental status.

Although limited data were reported regarding patient eligibility for thrombolysis, recent research suggests that EMSrecognised strokes are more likely to be prealerted to hospital ${ }^{29}$; are assessed more rapidly in the $\mathrm{ED}^{25}$; have faster door-to-CT times $^{20} 23$ and a greater likelihood of thrombolysis. ${ }^{20}$ Further research is needed to explore the impact of a missed prehospital diagnosis on eligibility for time-dependent stroke treatments and on patient outcomes.

There were a number of limitations of the studies included. The majority of studies involved the validation or performance of prehospital stroke screening tools, entailed specialist training, and were mainly undertaken in selected groups of patients with confirmed or suspected stroke/TIA. Therefore, screening might only have been completed in patients for whom the EMS clinician already had a high index of suspicion for stroke and their subsequent labelling of stroke was determined by a clinical protocol. It was not clear in any of the studies whether symptom data had been recorded by the EMS or whether symptoms had been completely missed by the EMS and only recorded in hospital. Fourteen of the 21 included studies were at high risk of selection bias mainly due to retrospective data collection, which may have resulted in not all relevant patient symptoms being recorded; the majority of studies were conducted in single EMS and hospital centres. Study quality and size varied considerably and there was a lack of reported data, limiting the generalisability of study findings. Only four studies reported stroke type. Of these, although three studies reported symptoms for all false negative patients, these were not reported by stroke subtype. It is unknown whether false negatives have the same proportion of $\mathrm{ICH}$ and ischaemic strokes as the standard stroke population, or whether there are factors to do with symptom recognition which made affect this balance, for example, change in conscious level. A further limitation of the review was the inclusion only of studies that were published in English. Although we contacted authors for further information, at the time of writing no responses had been received. Some studies were excluded where the characteristics of false negative patients and patients with stroke mimics were not reported separately. As this review focused on the emergency assessment of stroke patients in prehospital settings, there may be other studies not included in this review that have reported data on false negative stroke patients. Eleven further papers reported the numbers of false negative patients but very little other data. Previous research has highlighted the failure of studies to identify and report false negative stroke patients, 
particularly in studies which involve the selection and transportation of patients to specialist stroke centres. ${ }^{39}$ It is important that future research studies which include false negative patients report more detail about this population to further understand their characteristics, the symptoms they experienced and any impact on patient outcomes.

While it may not be possible for EMS personnel to identify all stroke patients without reducing specificity, ongoing research in selected patients is exploring the use of point-of-care diagnostics. A range of diagnostic techniques are currently in development, but none are currently used routinely in practice. ${ }^{40}$ Therefore, the recognition of suspected patients who had a stroke with the triaging of patients who present with stroke mimics and associated overuse of high priority EMS resources will continue to be challenging for the EMS.

\section{CONCLUSIONS}

Stroke presentations that are most frequently missed by the EMS commonly include symptoms of: speech problems, nausea/ vomiting, dizziness, changes in mental status and visual disturbance/ impairment. However, the addition of further symptoms to stroke screening tools would require evaluation of their sensitivity and specificity, any associated training needs, and the impact on EMS resource use. Despite the inclusion of speech symptoms in most prehospital screening tools, this symptom is often overlooked and the reasons for this may need to be explored further.

\section{Twitter Graham McClelland @mcclg}

Contributors SPJ: design and methodology, data collection, assessing risk of bias, data analysis, writing-up, full checking of final draft. JEB: design and methodology, writing-up, full checking of final draft. JMEG: design and methodology, assessing risk of bias, writing-up, full checking of final draft. CM: design and methodology, data collection, data analysis, assessing risk of bias, writing-up, full checking of final draft. GM: design and methodology, writing-up, full checking of final draft. CP: design and methodology, writing-up, full checking of final draft. CLW: design and methodology, writing-up, full checking of final draft.

Funding Funding for this review was received from the The University of Central Lancashire Research Institute For Global Health and Well-being (LIFE). JEB is supported by the Heart Foundation of Australia and the National Health and Medical Research Council (NHMRC) Prehospital Care Centre of Research Excellence.

Competing interests JEB has authored papers included in this systematic review. Patient consent for publication Not required.

Provenance and peer review Not commissioned; externally peer reviewed. Data availability statement All data relevant to the study are included in the article.

Supplemental material This content has been supplied by the author(s). It has not been vetted by BMJ Publishing Group Limited (BMJ) and may not have been peer-reviewed. Any opinions or recommendations discussed are solely those of the author(s) and are not endorsed by BMJ. BMJ disclaims all liability and responsibility arising from any reliance placed on the content. Where the content includes any translated material, BMJ does not warrant the accuracy and reliability of the translations (including but not limited to local regulations, clinical guidelines, terminology, drug names and drug dosages), and is not responsible for any error and/or omissions arising from translation and adaptation or otherwise.

Open access This is an open access article distributed in accordance with the Creative Commons Attribution Non Commercial (CC BY-NC 4.0) license, which permits others to distribute, remix, adapt, build upon this work non-commercially, and license their derivative works on different terms, provided the original work is properly cited, appropriate credit is given, any changes made indicated, and the use is non-commercial. See: http://creativecommons.org/licenses/by-nc/4.0/.

\section{ORCID iDs}

Stephanie P Jones http://orcid.org/0000-0001-9149-8606

Graham McClelland http://orcid.org/0000-0002-4502-5821

Chris I Price http://orcid.org/0000-0003-3566-3157

\section{REFERENCES}

1 World Health Organization. The world health report: 2002: reducing risks, promoting healthy life. World Health Organization, 2002.

2 Wardlaw JM, Murray V, Berge E, et al. Recombinant tissue plasminogen activator for acute ischaemic stroke: an updated systematic review and meta-analysis. Lancet 2012;379:2364-72.

3 Vidale S, Longoni M, Valvassori L, et al. Mechanical thrombectomy in strokes with large-vessel occlusion beyond 6 hours: a pooled analysis of randomized trials. J Clin Neurol 2018;14:407-12.

4 Adeoye 0, Lindsell C, Broderick J, et al. Emergency medical services use by stroke patients: a population-based study. Am J Emerg Med 2009;27:141-5.

5 Kothari RU, Pancioli A, Liu T, et al. Cincinnati prehospital stroke scale: reproducibility and validity. Ann Emerg Med 1999;33:373-8.

6 Zhelev Z, Walker G, Henschke N, et al. Prehospital stroke scales as screening tools for early identification of stroke and transient ischemic attack. Cochrane Database Syst Rev 2019;4:CD011427.

7 National Institute for Health and Care Excellence. Health and social care directorate quality Standards and indicators Briefing paper, 2015. Available: https://www.nice. org.uk/guidance/qs2/documents/stroke-quality-standards-update-qs-briefing-paper2 [Accessed 18 Dec 2018].

8 Sarraj A, Medrek S, Albright K, et al. Posterior circulation stroke is associated with prolonged door-to-needle time. Int I Stroke 2015;10:672-8.

9 Whiting PF, Rutjes AWS, Westwood ME, et al. QUADAS-2: a revised tool for the quality assessment of diagnostic accuracy studies. Ann Intern Med 2011;155:529-36

10 Jones SP, Price $\mathrm{Cl}$, McLelland $\mathrm{G}$. The characteristics of stroke patients inaccurately identified by the emergency medical services during prehospital assessment: a systematic review. PROSPERO 2019;CRD42019125170. Available: http://www.crd. york.ac.uk/PROSPERO/display_record.php?ID=CRD42019125170

11 Moher D, Liberati A, Tetzlaff J, et al. Preferred reporting items for systematic reviews and meta-analyses: the PRISMA statement. Ann Intern Med 2009;151:264-9.

12 Kidwell CS, Starkman S, Eckstein M, et al. Identifying stroke in the field. prospective validation of the Los Angeles prehospital stroke screen (LAPSS). Stroke 2000;31:71-6.

13 Ramanujam P, Guluma KZ, Castillo EM, et al. Accuracy of stroke recognition by emergency medical dispatchers and paramedics--San Diego experience. Prehosp Emerg Care 2008;12:307-13.

14 Frendl DM, Strauss DG, Underhill BK, et al. Lack of impact of paramedic training and use of the Cincinnati prehospital stroke scale on stroke patient identification and on-scene time. Stroke 2009;40:754-6.

15 Smith WS, Corry MD, Fazackerley J, et al. Improved paramedic sensitivity in identifying stroke victims in the prehospital setting. Prehosp Emerg Care 1999;3:207-10.

16 Studnek JR, Asimos A, Dodds J, et al. Assessing the validity of the Cincinnati prehospital stroke scale and the medic prehospital assessment for code stroke in an urban emergency medical services agency. Prehosp Emerg Care 2013;17:348-53.

17 Asimos AW, Ward S, Brice JH, et al. Out-Of-Hospital stroke screen accuracy in a state with an emergency medical services protocol for routing patients to acute stroke centers. Ann Emerg Med 2014;64:509-15.

18 Brandler ES, Sharma M, McCullough F, et al. Prehospital stroke identification: factors associated with diagnostic accuracy. J Stroke Cerebrovasc Dis 2015;24:2161-6.

19 McMullan JT, Katz B, Broderick J, et al. Prospective prehospital evaluation of the Cincinnati stroke triage assessment tool. Prehosp Emerg Care 2017;21:481-8.

20 Oostema JA, Konen J, Chassee T, et al. Clinical predictors of accurate prehospital stroke recognition. Stroke 2015;46:1513-7.

21 Fothergill RT, Williams J, Edwards MJ, et al. Does use of the recognition of stroke in the emergency room stroke assessment tool enhance stroke recognition by ambulance clinicians? Stroke 2013;44:3007-12.

22 Huwez F, Casswell EJ. FAST-AV or FAST-AB tool improves the sensitivity of fast screening for detection of posterior circulation strokes. Int I Stroke 2013;8:E3.

23 Sheppard JP, Mellor RM, Greenfield S, et al. The association between prehospital care and in-hospital treatment decisions in acute stroke: a cohort study. Emerg Med J 2015;32:93-9

24 Bray JE, Martin J, Cooper G, et al. Paramedic identification of stroke: community validation of the Melbourne ambulance stroke screen. Cerebrovasc Dis 2005;20:28-33.

25 Mosley I, Nicol M, Donnan G, et al. The impact of ambulance practice on acute stroke care. Stroke 2007:38:2765-70.

26 Bray JE, Coughlan K, Barger B, et al. Paramedic diagnosis of stroke: examining long-term use of the Melbourne ambulance stroke screen (mass) in the field. Stroke 2010;41:1363-6

27 Chen S, Sun H, Lei Y, et al. Validation of the Los Angeles pre-hospital stroke screen (LAPSS) in a Chinese urban emergency medical service population. PLoS One 2013;8:e70742.

28 Mingfeng $\mathrm{H}$, Zhixin W, Qihong G, et al. Validation of the use of the ROSIER scale in prehospital assessment of stroke. Ann Indian Acad Neurol 2012;15:191-5.

29 Andersson E, Bohlin L, Herlitz J, et al. Prehospital identification of patients with a final Hospital diagnosis of stroke. Prehosp Disaster Med 2018;33:63-70. 
30 Berglund A, Svensson L, Wahlgren N, et al. Face arm speech time test use in the prehospital setting, better in the ambulance than in the emergency medical communication center. Cerebrovasc Dis 2014;37:212-6.

31 Chenkin J, Gladstone DJ, Verbeek PR, et al. Predictive value of the Ontario prehospital stroke screening tool for the identification of patients with acute stroke. Prehosp Emerg Care 2009;13:153-9.

32 Jones SP, Carter B, Ford GA, et al. The identification of acute stroke: an analysis of emergency calls. Int J Stroke 2013;8:408-12.

33 Bray JE, O'Connell B, Gilligan A, et al. Is fast stroke smart? Do the content and language used in awareness campaigns describe the experience of stroke symptoms? Int J Stroke 2010;5:440-6.

$34 \mathrm{McClelland} \mathrm{G}$, Rodgers $\mathrm{H}$, Price $\mathrm{Cl}$. A survey of UK ambulance service stroke admission pathways. Int J Stroke 2018;13:3S.
35 Kerber KA, Brown DL, Lisabeth LD, et al. Stroke among patients with dizziness, vertigo, and imbalance in the emergency department: a population-based study. Stroke 2006;37:2484-7.

36 Aroor S, Singh R, Goldstein LB. BE-FAST (balance, eyes, face, arm, speech, time): reducing the proportion of strokes missed using the fast mnemonic. Stroke 2017;48:479-81.

37 Gulli G, Markus HS. The use of fast and ABCD2 scores in posterior circulation, compared with anterior circulation, stroke and transient ischemic attack. J Neurol Neurosurg Psychiatry 2012;83:228-9.

38 Pickham D, Valdez A, Demeestere J, et al. Prognostic value of BEFAST vs. fast to identify stroke in a prehospital setting. Prehosp Emerg Care 2019;23:195-200.

39 Price $\mathrm{Cl}$, Clement F, Gray J, et al. Systematic review of stroke thrombolysis service configuration. Expert Rev Neurother 2009;9:211-33.

40 Walsh KB. Non-Invasive sensor technology for prehospital stroke diagnosis: current status and future directions. Int J Stroke 2019;14:592-602. 